\title{
artigo
}

Veloso, L.P.; Barreiros, R.N.; Dutra, L.B.; Loura, T.Q.;

A terapia com laser de baixa potência como recurso coadjuvante no tratamento da artrite reumatóide - revisão integrativa

\section{A terapia com laser de baixa potência como recurso coadjuvante no tratamento da artrite reumatóide - revisão integrativa}

\author{
Low-level laser therapy as a supporting resource in the treatment of rheumatoid arthritis - integrative review \\ La terapia con láser de baja potencia como recurso de apoyo en el tratamiento de la artritis reumatoide - revisión integrativa
}

\begin{abstract}
RESUMO
Objetivo: Analisar as evidências do tratamento com laser de baixa potência aplicada em pacientes com artrite reumatoide. Métodos: Este estudo trata-se de uma revisão integrativa da literatura utilizando artigos científicos indexados nas bases de dados Pudmed e Biblioteca Virtual de Saúde (BVS) através de descritores relacionados a artrite reumatoide e laser de baixa potência, de acordo com os descritores em saúde. 0 rastreamento foi realizado em julho de 2020. Resultados: Os artigos obtidos foram publicados entre os anos 2010 à 2020, foram encontrados 266 artigos, após exclusão dos artigos que não se classificavam aos critérios de inclusão e os duplicados foram selecionados quatro. Constatou-se que a terapia com laser de baixa potência é um recurso terapêutico viável e promissor para os pacientes com artrite reumatoide. Em função do baixo número de publicações sobre o assunto, é notável a necessidade de mais pesquisas direcionadas à aplicabilidade do laser nas doenças reumáticas.

DESCRITORES: Bioestimulação a Laser; Artrite Reumatoide; Laser de baixa potência; Terapia com Luz de Baixa Intensidade.
\end{abstract}

\section{ABSTRACT}

Objective: To analyze the evidence of low-level laser treatment applied to patients with rheumatoid arthritis. Methods: This study is an integrative literature review using scientific articles indexed in the Pudmed and Virtual Health Library (VHL) databases through descriptors related to rheumatoid arthritis and low-level laser, according to the health descriptors. The screening was carried out in July 2020. Results: The articles obtained were published between the years 2010 to 2020, 266 articles were found, after the exclusion of articles that did not meet the inclusion criteria and four duplicates were selected. Low-level laser therapy was considered a viable and promising therapeutic resource for patients with rheumatoid arthritis. Due to the small number of publications on the subject, the need for more research directed to the applicability of laser in rheumatic diseases is notable.

DESCRIPTORS: Laser biostimulation; Rheumatoid arthritis; Low power laser; Low intensity light therapy.

\section{RESUMEN}

Objetivo: Analizar la evidencia del tratamiento con láser de baja intensidad aplicado a pacientes con artritis reumatoide. Métodos: Este estudio es una revisión integradora de la literatura utilizando artículos científicos indexados en las bases de datos de Pudmed y Biblioteca Virtual en Salud (BVS) a través de descriptores relacionados con la artritis reumatoide y láser de baja intensidad, según los descriptores de salud. El cribado se realizó en julio de 2020. Resultados: Los artículos obtenidos fueron publicados entre los años 2010 a 2020, se encontraron 266 artículos, luego de la exclusión de los artículos que no cumplieron con los criterios de inclusión y se seleccionaron cuatro duplicados. La terapia con láser de baja intensidad se consideró un recurso terapéutico viable y prometedor para los pacientes con artritis reumatoide. Debido al escaso número de publicaciones sobre el tema, es notable la necesidad de más investigación dirigida a la aplicabilidad del láser en enfermedades reumáticas. DESCRIPTORES: Bioestimulación láser; Artritis reumatoide; Láser de baja potencia; Terapia de luz de baja intensidad.

RECEBIDO EM: 09/02/2021 APROVADO EM: 17/05/2021

\section{Luiza Pokrajac Veloso}

Fisioterapeuta, Pós graduada em Acupuntura (Faculdade São Judas Tadeu), Fisioterapia em Oncologia (Instituto Nacional do Câncer - INCA) e em Pediatria e Neonatologia (Unigranrio).

ORCID: 0000-0003-0214-0739 


\section{Raphael Neves Barreiros}

Enfermeiro. Mestre em Enfermagem pela Universidade Federal do Estado do Rio de Janeiro (UNIRIO); Pós Graduado em Acupuntura (UNIBAHIA).

ORCID: 0000-0002-0272-3187

\section{Luana Borges Dutra}

Enfermeira. Mestre em Enfermagem pela Universidade Federal do Estado do Rio de Janeiro (UNIRIO); Pós Graduada em Acupuntura (UNIBAHIA).

ORCID: 0000-0002-6132-3255

\section{Thiago Quinellato Louro}

Enfermeiro, Pós Doutor em Enfermagem, Doutor em Ciências - PPGENFBIO/UNIRIO. Professor Adjunto II do Departamento de Enfermagem do Instituto de Humanidades em Saúde da Universidade Federal Fluminense (UFF). Coordenador do Grupo de Pesquisa: Laboratório de Pesquisa e Experimentação para o Ensino e Cuidado em Enfermagem - L@PETECEnf / UFF.

ORCID: 0000-0001-8371-628X

\section{INTRODUÇÃO}

A artrite reumatoide (AR) é uma doença autoimune, crônica, progressiva, sistêmica e de ampla prevalência. Essa condição clínica está associada a níveis álgicos elevados, comprometimento funcional, e distúrbios psicoemocionais que afetam a qualidade de vida e podem levar a incapacidade e ao possível afastamento das atividades laborais ${ }^{1,2}$. Tendo em vista o crescimento demográfico ocidental de indivíduos com mais de 60 anos, em aproximadamente $30 \%$ dos casos, a morbidade, mortalidade e incapacidade associadas à $\mathrm{AR}$ aumentarão ${ }^{1}$.

A AR compromete basicamente as articulações de maneira generalizada, simétrica, porém é caracterizada pelo processo inflamatório persistente nas membranas sinoviais, atingindo principalmente as articulações do tipo diartrodiais, ou seja, sinoviais. Essas são articulações móveis, formadas por cartilagem hialina, no encontro de dois ossos com líquido sinovial (água com proteoglicanos, glicosaminoglicanos similar ao plasma) mantido por uma membrana sinovial formada por fibrocartilagem ${ }^{3}$.

Um dos mecanismos patogenéticos envolvidos na inflamação imune progressiva e descontrolada da membrana sinovial nas dobradiças é o desequilíbrio entre a síntese de citocinas pró-inflamatórias e anti-inflamatórias, com predominância da primeira sobre a segunda ${ }^{4}$.

Esse processo está associado à migração de fagócitos ativados e leucócitos para o tecido sinovial e periarticular ${ }^{5}$. Durante a fagocitose, neutrófilos e macrófagos ativados geram espécies reativas de oxigênio, destruições de lipídios, das proteínas, do ácido hialurônico (importante glicosaminoglicano na constituição da articulação) e da cartilagem ${ }^{6}$. A destruição de lipídios produz a peroxidação, que se mostrou elevada no plasma e no líquido sinovial desses pacientes6; além de levar ao comprometimento dos sistemas de defesa antioxidante, em favor da peroxidação lipídica, causando danos aos tecidos?.

\section{A artrite}

reumatoide (AR)

é uma doença

autoimune, crônica,

progressiva,

sistêmica e de ampla

prevalência.
As espécies reativas de nitrogênio também desempenham um papel nas reações oxidativas mediadas por fagócitos nos locais de inflamação ${ }^{8}$. Elas incluem óxido nítrico, peroxinitrito, nitrito e nitrato. Um estudo prévio mostrou aumento dos níveis séricos de óxido nítrico em pacientes com AR?.

De etiologia desconhecida, a AR é caracterizada pela inflamação articular e pela formação de um tecido inflamatório e invasivo conhecido como pannus reumatoide, que eventualmente leva à destruição das articulações ${ }^{10}$. As citocinas e outros mediadores pró-inflamatórios, como o fator de necrose tumoral (TNF$-\alpha)$, IL - $1 \beta$ e IL - 6, desempenham papéis importantes na inflamação e nas degenerações progressivas da cartilagem articular e do osso ${ }^{11,12}$. O dano persistente resultará inevitavelmente à incapacidade, pois a maioria dos danos nas articulações são irreversíveis ${ }^{13,14}$.

A citocina que se encontra na sinóvia reumatoide em maior quantidade é a IL - 6. Em resposta às condições inflamatórias agudas e crônicas nesta patologia reumatológica, a IL-6 é responsável por estimular os hepatócitos a sintetizar e secretar proteínas de fase aguda, como a proteína $\mathrm{C}$ reativa (PCR) e o fibrinogênio. Em estudos prévios, a PCR mostrou-se elevada em comparação ao grupo controle, assim como a taxa de sedimentação de eritrócitos (VHS) na presença de fibrinogênio, ambos na fase aguda $e$ em pacientes com $\mathrm{AR}^{15,16}$. 


\section{artigo}

Veloso, L.P.; Barreiros, R.N.; Dutra, L.B.; Loura, T.Q.;

A terapia com laser de baixa potência como recurso coadjuvante no tratamento da artrite reumatóide - revisão integrativa

O tratamento da AR visa prevenir as deformidades articulares e sua funcionalidade, retardar a progressão da doença, e minimizar os sintomas ${ }^{17}$. Os anti-inflamatórios não esteroides (AINE) são os mais prescritos para as artralgias, edemas, e rigidez matinal, porém o desencadeamento de úlceras gastrointestinais e morbidades renais são alguns dos seus efeitos adversos bem estabelecidos ${ }^{16}$.

O metotrexato é a droga antirreumática de primeira linha direcionada para inibir a atividade ou remissão da doença $^{18}$. Em contrapartida, a toxicidade imbenefícios. Em pesquisas recentes, foi relatado que toxicidade sutil ocorre em cerca de $60 \%$ dos pacientes, e aproximadamente $7 \%$ a $30 \%$ dos pacientes descontinuam a terapia com metotrexato no primeiro ano de tratamento ${ }^{19,20}$. As toxicidades causadas por medicamentos afetam muito os resultados terapêuticos desses pacientes, e pode ser um desafio para os idosos ${ }^{3}$.

A qualidade de vida ( $Q V)$ é uma característica integrante do funcionamento físico, psicológico, emocional e social do paciente, com base em sua percepção subjetiva ${ }^{1}$. Os objetivos esperados pelas terapias modernas e reabilitação desse público são a analgesia, prevenção e retorno da pede muitos pacientes de obterem seus

função das articulações comprometidas e manter a capacidade de autocuidado ${ }^{8}$.

A QV em pacientes idosos pode ser otimizada com medicina complementar e alternativa (MCA). O uso da MCA aumentou em ambientes convencionais de assistência médica, pelo receio dos efeitos adversos medicamentosos e o desejo do alívio dos sintomas. No entanto, muitas modalidades da MCA não têm evidências científicas para apoiar sua eficácia e segurança ${ }^{20}$.

$\mathrm{O}$ tratamento com acupuntura a laser tornou-se amplamente aceito e atualmente é oferecido como uma terapia poderosa e eficaz. A fotobiomodulação aplicada aos acupontos gera efeito fisiológico a nível celular desencadeando analgesia, modulação do processo inflamatório e do estresse oxidativo, ativação da angiogênese, e melhora do reparo tecidual ${ }^{21,22,23,24,25}$. Embora a literatura atual mostre efeitos positivos a favor da terapia a laser no tratamento de vários tipos de inflamações articulares, poucos autores pesquisaram o uso do laser no tratamento da $\mathrm{AR}^{24,25,26,27}$.

Outro estudo relata que os pacientes com AR apresentam altos níveis plasmáticos de leptina, o que sugere processo inflamatório ativo, pois a leptina pode atuar como uma citocina pró-inflamatória. Neste mesmo estudo, é demonstrado que a terapia com laser intravenoso de baixa

Figura 1. Fluxograma explicativo da seleção dos artigos sobre fotobiomodulação nas implicações clínicas da artrite reumatoide.

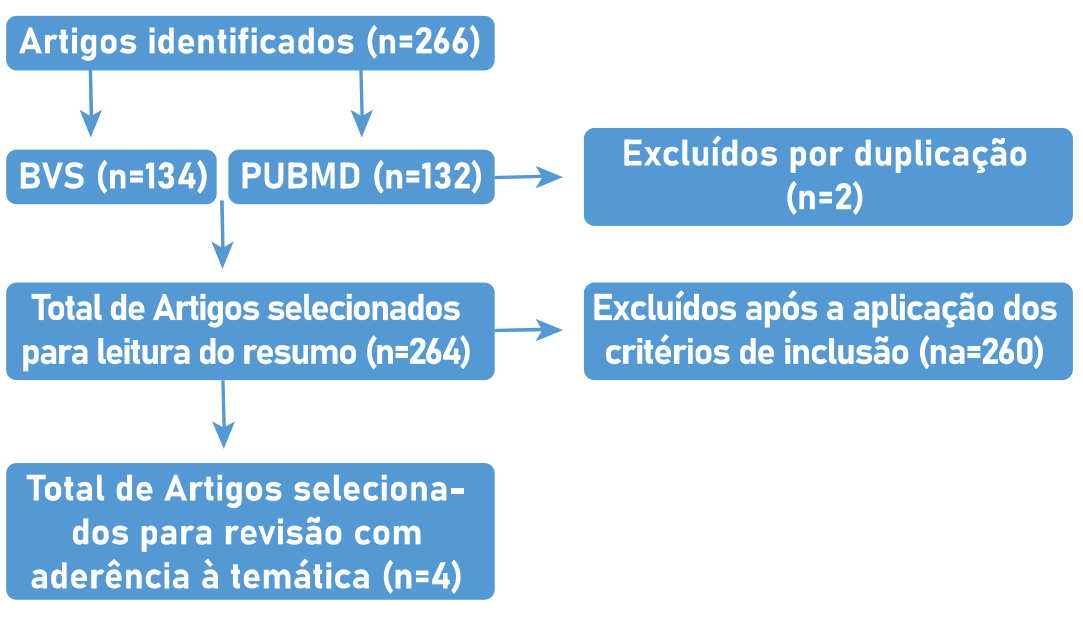

Fonte: autor. intensidade pode normalizar os níveis de leptina plasmática e trazer qualidade de vida a esses pacientes ${ }^{3}$.

O objetivo do presente estudo foi levantar os artigos publicados nos últimos 10 anos e demonstrar os resultados clínicos do laser de baixa potência aplicada aos pacientes com artrite reumatoide.

\section{MÉTODO}

O presente estudo trata-se de uma revisão integrativa. As etapas desta pesquisa foram: realização da amostragem, classificação dos estudos, definição das informações selecionadas das publicações dos resultados deste trabalho. A coleta de dados foi realizada em julho de 2020, nas bases de dados Biblioteca Virtual de Saúde e Pubmed. Os descritores utilizados, de forma ordenada, foram: Photobiomodulation; photobiomodulation therapy; "low-level ligth therapy" OR "low-level laser therapy" OR "laser therapy" OR LLLT) AND rheumatoid arthritis, com base na consulta no Desc e interligados pelos operadores booleanos 'AND' e 'OR'.

Os critérios de inclusão adotados foram os seguintes: artigos publicados nos últimos dez anos, controlado, randomizados, duplo cego, realizados em seres humanos, nos idiomas português, inglês, espanhol e russo, cujos títulos e/ou resumos estivessem relacionados à temática desta pesquisa, com acesso aberto, disponibilizados na integra.

Os critérios de exclusão foram todos os estudos que não se enquadram nos critérios de inclusão, as publicações duplicadas, e as pesquisas que não contemplam o objetivo desta revisão.

O rastreamento foi realizado no dia 04 de julho de 2020 e foram achados 266 artigos, sendo 134 artigos da BVS e 132 do Pubmed, contudo, após a filtragem destas bases de dados restaram seis artigos na BVS e cinco no Pubmed, sendo dois em duplicidade e cinco que não corresponderam à temática norteadora do estudo em tela no título e/ou resumo, totalizando em quatro artigos para construção desta revisão. 


\section{RESULTADOS}

Os conteúdos extraídos dos artigos selecionados foram organizados na planilha abaixo:

\section{DISCUSSÃO}

AR é a doença mais frequente e a mais grave que domina a prática reumatológica.
Adly et al afirmam que a prevalência aumenta com a idade, e que é provável que a morbidade, mortalidade e incapacidade associadas à $\mathrm{AR}$ aumentem entre os idosos no futuro, pois no ocidente o número de indivíduos acima de 60 anos é crescente e em torno de $30 \%$ dos casos de AR ocorrem nesta faixa etária ${ }^{1}$.

O tratamento da AR é direcionado a inibir a atividade da doença e a promo- ver sua remissão pelo uso de drogas antirreumáticas modificadoras da doença como o metotrexato1. Os anti-inflamatórios usados com mais frequência na AR são os não esteroides (AINEs) que com seu uso crônico levam a efeitos colaterais como úlceras gastrointestinais e morbidade renal ${ }^{1,20}$.

Investigamos o uso da FBM como tratamento coadjuvante na AR e observamos

Tabela 1: Características dos estudos incluídos no estudo. Rio de Janeiro, 2020.

\begin{tabular}{|c|c|c|c|c|c|}
\hline $\begin{array}{l}\text { BASE DE } \\
\text { DADOS }\end{array}$ & $\begin{array}{l}\text { PAÍS DE } \\
\text { ORIGEM }\end{array}$ & TÍTULO & $\begin{array}{l}\text { TIPO DE } \\
\text { ESTUDO }\end{array}$ & AUTORES & $\begin{array}{l}\text { PERIÓDICOS E ANO DE } \\
\text { PUBLICAÇÃO }\end{array}$ \\
\hline $\begin{array}{l}\text { BVS e } \\
\text { PUBMED }\end{array}$ & Inglaterra & $\begin{array}{c}\text { Laser acupuncture versus reflexology } \\
\text { therapy in elderly with rheumatoid } \\
\text { arthritis. }\end{array}$ & $\begin{array}{l}\text { Ensaio } \\
\text { Clínico }\end{array}$ & $\begin{array}{l}\text { Adly, Afnan Sedky; Adly, Aya } \\
\text { Sedky; Adly, Mahmoud Sedky; } \\
\text { Serry, Zahra M H }\end{array}$ & $\begin{array}{l}\text { Laser in medical } \\
\text { Science / } 2017\end{array}$ \\
\hline BVS & Brasil & $\begin{array}{l}\text { Assessment of the effectiveness of } \\
\text { low-level laser therapy on the hands } \\
\text { of patients with rheumatoid arthritis: } \\
\text { a randomized double-blind controlled } \\
\text { trial. }\end{array}$ & $\begin{array}{l}\text { Ensaio } \\
\text { Clínico }\end{array}$ & $\begin{array}{l}\text { Meireles, Sandra Mara; Jones, } \\
\text { Anamaria; Jennings, Fábio; } \\
\text { Suda, Alina Lica; Parizotto, } \\
\text { Nivaldo Antônio; Natour, Jamil. }\end{array}$ & $\begin{array}{l}\text { Clinical Rheumatology } \\
\qquad / 2010\end{array}$ \\
\hline BVS & Rússia & $\begin{array}{l}\text { The influence of intravenous laser irra- } \\
\text { diation of the blood on the dynamics of } \\
\text { leptin levels and the quality of life of the } \\
\text { patients presenting with rheumatoid } \\
\text { arthritis. }\end{array}$ & $\begin{array}{l}\text { Ensaio } \\
\text { Clínico }\end{array}$ & $\begin{array}{c}\text { Starodubtseva, I A; Vasilieva, L } \\
\text { V; Nikitin, A V }\end{array}$ & $\begin{array}{l}\text { Vopr Kurortol Fizioter } \\
\text { Lech Fiz Kult / } 2015\end{array}$ \\
\hline $\begin{array}{l}\text { BVS e } \\
\text { PUBMED }\end{array}$ & Egito & $\begin{array}{c}\text { Therapeutic antioxidant and anti-in- } \\
\text { flammatory effects of laser acupunc- } \\
\text { ture on patients with rheumatoid } \\
\text { arthritis. }\end{array}$ & $\begin{array}{l}\text { Ensaio } \\
\text { Clínico }\end{array}$ & $\begin{array}{l}\text { Attia, Atef M M; Ibrahim, Fatma } \\
\text { A A; Abd El-Latif, Noha A; Aziz, } \\
\text { Samir W; Elwan, Azhar M; Abdel } \\
\text { Aziz, Abdel Aziz A; Elgendy, } \\
\text { Aliaa; Elgengehy, Fatema T. }\end{array}$ & $\begin{array}{l}\text { Lasers in Surgery and } \\
\text { Medicine / } 2016\end{array}$ \\
\hline Fonte: a & & & & & \\
\hline
\end{tabular}

Tabela 2: Principais experiências e resultados dos artigos encontrados. Rio de Janeiro 2020.

TÍTULO DO ARTIGO

Laser acupuncture versus reflexology therapy in elderly with rheumatoid arthritis.

Assessment of the effectiveness of low-level laser therapy on the hands of patients with rheumatoid arthritis: a randomized double-blind controlled trial.

\section{EXPERIÊNCIAS}

PRINCIPAIS RESULTADOS

Determinar e comparar a eficácia da acupuntura a laser versus reflexologia em idosos com artrite reumatoide. Na terapia com acupuntura a laser utilizou-se os seguintes parâmetros de aplicação: $904 \mathrm{~nm}$, área de feixe de $1 \mathrm{~cm}(2)$, potência 100 $\mathrm{mW}$, densidade de potência $100 \mathrm{~mW} / \mathrm{cm} 2$, dosagem de energia $4 \mathrm{~J}$, densidade de energia $4 \mathrm{~J} / \mathrm{cm}$ 2, tempo de irradiação 40 segundos e frequência $100.000 \mathrm{~Hz}$, já na técnica de reflexologia utilizou-se as mãos do terapeuta para estimulação dos pontos específicos nos pés.

Avaliar a efetividade da terapia a laser de baixo nível na redução da dor e melhora da função nas mãos de pacientes com artrite reumatoide
A aplicabilidade do laser em acupontos em idosos com artrite reumatoide mostrou-se eficaz e apresentou os resultados superiores acerca do aumento de ATP mitocondrial, da angiogênese, e amplitude de movimento nas articulações de tornozelos e punhos, redução do estresse oxidativo (IL-6 e MDA), dos questionários RAQoL e HAQe; em relação a terapia de reflexologia.

O estudo demonstrou que não houve resultados relevantes na analgesia e na função das mãos de paciente com artrite reumatoide com a terapia a laser de baixo nível com os parâmetros aplicados nesta pesquisa. 


\section{artigo}

Veloso, L.P.; Barreiros, R.N.; Dutra, L.B.; Loura, T.Q.;

A terapia com laser de baixa potência como recurso coadjuvante no tratamento da artrite reumatóide - revisão integrativa

The influence of intravenous laser irradiation of the blood on the dynamics of leptin levels and the quality of life of the patients presenting with rheumatoid arthritis.
Estudar o efeito da laserterapia intravenosa, utilizando o VLOK como instrumento de aplicação da técnica na dinâmica do conteúdo plasmático de leptina, bem como na qualidade de vida de pacientes com AR.
O curso da laserterapia intravenosa foi realizado utilizando o aparelho Matrix-VLOK (Ma-Trix, Rússia) e foi notada a redução dos níveis plasmáticos de leptina e aumento da qualidade de vida dos pacientes do grupo laser, demonstrando resultado superior ao grupo controle.

Constatou-se que os efeitos terapêuticos da acupuntura a laser podem ocorrer através do alívio do estresse oxidativo e da modulação do processo inflamatório, além de melhorar o status metabólico antioxidante e energético em pacientes com artrite reumatoide. nos artigos aqui analisado que o parâmetro mais importante na definição do regime ideal de distribuição de luz é a densidade de energia ${ }^{1}$. A FBM estimula a produção de ATP, o potencial de membrana mitocondrial e a proliferação celular. A enzima citocromo $\mathrm{C}$ oxidase, também conhecida como unidade 4 da cadeia respiratório localizada na membrana mitocondrial interna, é um importante cromóforo ${ }^{28,29}$. Essa proteína possui bandas de absorção que se estendem da luz vermelha ao infravermelho próximo. Após a absorção da luz a cadeia de transporte de elétrons é estimulada, induzindo a um aumento na taxa de respiração celular e metabolismo celular, resultando no aumento da produção de ATP e síntese de DNA e RNA, proporcionando a melhoria da atividade de nutrição e oxigenação como um todo ${ }^{1}$.

O efeito celular primário do laser é aumentar a atividade enzimática, e deve durar um tempo suficiente para ter um efeito real no metabolismo celular. Como esclarecido acima, a literatura indica que o uso do laser é capaz de estimular processos biológicos intrínsecos relacionados ao ciclo celular, como proliferação, diferenciação e crescimento, o que é benéfico na atuação conservadora desta terapia em pacientes com atrite reumatoide ${ }^{23,25}$.

Os quatro estudos consultados mostraram diferentes dosimetrias, locais, formas e tempo de aplicação do laser, assim como o comprimento de onda, e com propósi-

\section{A FBM estimula}

a produção de

ATP, o potencial

de membrana

mitocondrial e a

proliferação celular.

A enzima citocromo

C oxidase, também

conhecida como

unidade $4 \mathrm{da}$

cadeia respiratório

localizada na

membrana

mitocondrial interna,

é um importante

cromóforo. tos terapêuticos distintos. No caso da AR sabe-se que a aplicação com contato permite maior penetração do laser, e a aplicação mais importante é na linha articular, uma vez que a $A R$ afeta principalmente as membranas sinoviais. Segundo a World Association for Photobiomodulation Therapy para o comprimento de onda 780-860 nm a energia aplicada deverá variar entre 1-16 Joules e o tempo de 20-300 segundos, já o comprimento de onda 904 $\mathrm{nm}$ a energia aplicada deverá variar entre 1-6 Joules e o tempo de 30-600 segundos, essas doses recomendadas são para os tipos de pele branca / caucasiana com base em resultados de ensaios clínicos ${ }^{30}$.

Ainda acerca da cronometria da aplicação, quando os fótons são absorvidos pela enzima citocromo $c$ oxidase levam a dissociação da molécula inibidora $\mathrm{ON}$ dos locais de ligação de $\mathrm{O} 2$ na enzima, aumentando assim a atividade da enzima na respiração celular. Se o efeito celular primário do laser é realmente aumentar a atividade de uma enzima, faz sentido supor que esse aumento da atividade deva durar um tempo suficiente para ter um efeito real no metabolismo celular. Isso explica alguns estudos com resultados negativos acerca da $\mathrm{FBM}^{25}$

O número de pacientes analisados nos artigos variou entre 30-132, sendo o número maior de mulheres, por conta de sua maior incidência neste gênero, a idade variou entre 18-70 entre os quatro estudos, e 
somente dois estudos relataram a calibração dos aparelhos.

Dois dos artigos analisados fizeram referência à raça, porém nenhum deles chegaram até o final de sua pesquisa com pacientes da raça negra no grupo controle. Seria uma comparação interessante, uma vez a potência inicial do feixe de laser é parcialmente perdida devido à absorção pela melanina ${ }^{3}$.

O tempo de permanecia da doença variou entre os estudos e alguns não apontaram esse dado que pode interferir claramente nos resultados acerca da FBM $\mathrm{Nem}$ todos os artigos aqui analisados citaram ou excluíram pacientes tabagistas de suas pesquisas, o que implica na labilidade dos resultados da FBM, tendo em vista que o tabagismo é considerado um dos fatores de risco mais bem estabelecidos para $\mathrm{AR}$, pois este hábito desencadeia modificações morfofuncionais no organismo além de desenvolver comorbidades e outras doenças, o que pode agravar ainda mais o quadro artrítico. A IL6 é a principal responsável pela destruição progressiva da cartilagem articular e do osso, pois é a citocina em maior quantidade encontrada na sinóvia reumatoide ${ }^{1}$.

$\mathrm{Na}$ patogenética envolvida na AR verificou-se que há um processo inflamatório progressivo e descontrolado da membrana sinovial nas articulações, onde existe um desequilíbrio entre a síntese de citosina pró-inflamatórias e anti-inflamatória, com predominância da primeira sobre a segunda. A FBM pode ser um recurso interessante, pois apresenta algumas evidências na diminuição de TNF-alfa e modulação do processo inflamatório, fatores envolvidos na causa das deformidades articulares. Outro estudo constatou que pacientes com AR apresentam aumento nos níveis plasmáticos de leptina, o que aumenta o processo inflamatório ${ }^{30}$. Porém, constataram o tratamento combinado por irradiação com laser intravenoso de baixa intensidade, os níveis de leptina são normalizados suprimindo o processo inflamatório e contribui para a melhora significativa da $\mathrm{QV}^{28}$.

Os quatro artigos analisados nesta revisão avaliaram, utilizando questionários e outras ferramentas quantitativas, distintas variáveis como os marcadores inflamatórios, oxidantes, antioxidantes, a dor, a rigidez matinal, a destreza manual, a capacidade funcional, a força muscular, a amplitude de movimento, o edema e a qualidade de vida dos pacientes com AR, lançando mão de aparelhos, parâmetros dosimétricos e formas de aplicabilidade da FBM heterogêneos. Somente um artigo não constatou resultados que fossem estatisticamente significativos, mas foi concluído neste último a necessidade de mais estudos com dosimetria padronizada para que o efeito real da fotobiomodulação seja esclarecido e bem indicado:

Dos indicadores avaliados nos estudos, os que tiveram maior evidência de resultados positivos foram a redução dos marcadores inflamatório $\mathrm{e}$ antioxidante. Este decréscimo favorece à normalização do funcionamento celular, a normalização da produção de ATP, a melhora da fun- ção pelo efeito analgésico, melhor qualidade de vida e autonomia. Faz-se mister pontuar que evidências atuais mostram que o diagnóstico precoce e a abordagem terapêutica convencional imediata pode retardar ou evitar a progressão da doença, permitindo que o paciente retorne às atividades a curto prazo e de forma mais produtiva.

\section{CONCLUSÃO}

A FBM é um recurso terapêutico viável e promissor para os pacientes com $A R$, pois além de sua aplicação ser rápida, indolor, atérmica, algumas evidências demonstraram que a FBM é eficaz na inibição da maioria dos principais fatores envolvidos na patogênese desta doença.

Como não há evidencias que apontem efeitos adversos acerca da FBM, esta terapia representa uma grande vantagem sobre o tratamento convencional dos sintomas da AR com o uso de AINES, pois melhora o status metabólico antioxidante e energético reduzindo desta forma a fadiga, modula a nocicepção minimizando a dor tornando-se uma alternativa não medicamentosa para o manejo da dor crônica, e consequentemente devolve este indivíduo às atividades laborais e recreativas.

Cabe ressaltar que mais estudos, com coerência e padronização dosimétricas, são necessários com o propósito de clarear os reais efeitos positivos da FBM, assim como sua indicação de forma assertiva, para que mais indivíduos se beneficiem desta terapia de fácil acessibilidade.

\section{REFERÊNCIAS}

1.Adly, AS, Adly, SA, Adly, MS, Serry, ZMH. Laser acupuncture versus reflexology therapy in elderly with rheumatoid arthritis. Lasers Med Sci. 2007 Jul;32(5):1097-1103. doi: 10.1007/ s10103-017-2213-y. Epub 2017 Apr 27. PMID: 28451815.

2. Starodubtseva, I A; Vasilieva, L V; Nikitin, A V. The influence of intravenous laser irradiation of the blood on the dynamics of leptin levels and the quality of life of the patients presenting with rheumatoid arthritis. Vopr Kurortol Fizioter Lech Fiz Kult; 92(3): 11-13, 2015. DOI: 10.17116 / kurort2015311-13.

3. Meireles SM, Jones A, Jennings F, Suda AL, Parizotto NA, Na- tour J. Assessment of the effectiveness of low-level laser therapy on the hands of patients with rheumatoid arthritis: a randomized double-blind controlled trial. Clin Rheumatol. 2010 May;29(5):501-9. doi: 10.1007/s10067-009-1347-0. Epub 2010 Jan 16. PMID: 20082104.

4. Oliveira $P$, Santos $A A$, Rodrigues $T$, Tim CR, Pinto KZ, Magri AM, Fernandes KR, Mattiello SM, Parizotto NA, Anibal FF, Rennó AC. Effects of phototherapy on cartilage structure and inflammatory markers in an experimental model of osteoarthritis. J Biomed Opt. 2013 Dec;18(12):128004. doi: 10.1117/1. 


\section{artigo}

Veloso, L.P.; Barreiros, R.N.; Dutra, L.B.; Loura, T.Q.;

A terapia com laser de baixa potência como recurso coadjuvante no tratamento da artrite reumatóide - revisão integrativa

\section{REFERÊNCIAS}

JBO.18.12.128004. PMID: 24343447.

5. Sarban S, Kocyigit A, Yazar M, Isikan UE. Plasma totalantioxidant capacity, lipid peroxidation, and erythrocyteantioxidant enzyme activities in patients with rheumatoidarthritis and osteoarthritis. Clin Biochem 2005;38:981-986.

6. Walwadkar SD, Suryakar AN, Katkam RV, Kumbar KM,Ankush RD. Oxidative stress and calcium-phosphorus levelsin rheumatoid arthritis. Indian J Clin Biochem 2006;21(2):134-137.

7. Ozkan Y, Yardym-Akaydyn S, Sepici A, Keskin E, Sepici V,Simsek B. Oxidative status in rheumatoid arthritis. ClinRheumatol 2007;26(1):64-68.

8. Pacher P, Beckman JS, Liaudet L. Nitric oxide andperoxynitrite in health and disease. Physiol Rev 2007;87(1):315-424.

9. Eiserich JP, Hristova $M$, Cross $C E$, Jones $A D$, Freeman $B A, H a l-$ liwell $B$, van der Vliet $A$. Formation of nitric oxide-derivedinflammatory oxidants by myeloperoxidase in neutrophils. Nature 1998;391(6665):393-397.

10. Ekambaram S, Perumal SS, Subramanian V. Evaluation ofantiarthritic activity of strychnos potatorum Linn seeds inFreund's adjuvant induced arthritic rat model. BMC Comple-ment Altern Med 2010;13(10):1-9.

11. Vervoordeldonk MJ, Tak PP. Cytokines in rheumatoidarthritis. Curr Rheumatol Reports 2002;4(3):208-217.12. Kamanli A, Naziroglu M, Aydile K, Hacievliyagil C. Plasmalipid peroxidation and antioxidant levels in patients withrheumatoid arthritis. Cell Biochem Funct 2004;22:53-57.

12. Yildirim K, Karatay S, Melioglu M, Gureser G, Ugur M, SenelK. Association between acute phase reactant levels anddisease activity score (DAS28) in patients with rheumatoidarthritis. Ann Clin Labor Sci 2004;34(4):423-426.

13. Ngian GS. Rheumatoid arthritis. Aust Fam Physician 2010;39(9):626-628.

14. Berard A, Solomon DH, Avorn J. Patterns of drug use inrheumatoid arthritis. J Rheumatol 2000;27(7):1648-1655.

15. Carteron NL. Cytokines in rheumatoid arthritis: Trials andtribulations. Mol Med Today 2000;6:315-323.

16. Singh G. Recent considerations in nonsteroidal anti-inflam-matory drug gastropathy. Am J Med 1998;105:31-38.

17. Jazayeri JA, Carrol GJ, Vernallis AB. Interleukin-6 subfam-ily cytokines and rheumatoid arthritis: Role of antagonists.Int Immunopharmacol 2010;10:1-8.

18. Emery $P$, Seto $Y$. Role of biologics in early arthritis. ExpRheumatol 2010;21(Suppl 30):191-194.

19. Sakamoto C, Soen S. Efficacy and safety of the selectivecyclooxygenase- 2 inhibitor celecoxib in the treatment ofrheumatoid arthritis and osteoarthritis in Japan. Digestion2011;83(1-2):108-123

20. rnst E, Fugh-Berman A (2002) Complementary and alternativemedicine: what is it all about? Occup Environ Med 59(2):140144.
21. Shea $B$, Swinden MV, Tanjong Ghogomu E, Ortiz Z, KatchamartW, Rader T, et al. (2013) Folic acidand folinic acid for reducing side effects in patients receiving meth-otrexate for rheumatoid arthritis. Cochrane Database Syst Rev 56.

22. Flores Luna GL, de Andrade ALM, Brassolatti P, Bossini PS, Anibal FF, Parizotto NA, Leal ÂMO. Biphasic Dose/Response of Photobiomodulation Therapy on Culture of Human Fibroblasts. Photobiomodul Photomed Laser Surg. 2020 Jul;38(7):413-418. doi: 10.1089/photob.2019.4729. Epub 2020 Mar 24. PMID: 32208063 .

23. Ferraresi C, Kaippert B, Avci P, Huang YY, de Sousa MV, Bagnato VS, Parizotto NA, Hamblin MR. Low-level laser (light) therapy increases mitochondrial membrane potential and ATP synthesis in C2C12 myotubes with a peak response at 3-6 h. Photochem Photobiol. 2015 Mar-Apr;91(2):411-6. doi: 10.1111/php.12397. Epub 2014 Dec 30. PMID: 25443662; PMCID: PMC4355185.

24. Ferraresi C, de Sousa MV, Huang YY, Bagnato VS, Parizotto NA, Hamblin MR. Time response of increases in ATP and muscle resistance to fatigue after low-level laser (light) therapy (LLLT) in mice. Lasers Med Sci. 2015 May;30(4):1259-67. doi: 10.1007/ s10103-015-1723-8. Epub 2015 Feb 21. PMID: 25700769.

25. Hamblin MR. Mechanisms and applications of the anti-inflammatory effects of photobiomodulation. AIMS Biophys. 2017;4(3):337-361. doi: 10.3934/biophy.2017.3.337. Epub 2017 May 19. PMID: 28748217; PMCID: PMC5523874.

26. Hu W-L, Chang $C-H$, Hung Y-C, Tseng Y-J, Hung I-L, Hsu S-F(2014) Laser acupuncture therapy in patients with treatment-resistant temporomandibular disorders. PLoS One 9(10):e110528.

27. Brosseau L, Welch V, Wells GA, de Bie R, Gam A, Harman K,Morin M, Shea B, Tugwell P (2005) Low level laser therapy(Classes I, II and III) for treating rheumatoid arthritis. TheCochrane Library.

28. Ives ACA, de Carvalho PTC, Parente M, Xavier M, Frigo L,Aimbire F, ECPL J, Albertini R (2013) Low-level laser therapy indifferent stages of rheumatoid arthritis: a histological study. LasersMed Sci 28(2):529-536.

29. Attia AM, Ibrahim FA, Abd El-Latif NA, Aziz SW, Elwan AM,Aziz A, Aziz A, Elgendy A, Elgengehy FT (2016) Therapeuticantioxidant and anti-inflammatory effects of laser acupuncture onpatients with rheumatoid arthritis. Lasers in surgery and medicine

30. WALT. Word Association for Lasertherapy. Dosage Recommendations, WALT [internet] 2010. Disponivel em: <https:// waltza.co.za/documentation-links/recommendations/dosage-recommendations/>.

31. Burduli NN, Burduli NM. [The influence of intravenous laser irradiation of the blood on the dynamics of leptin levels and the quality of life of the patients presenting with rheumatoid arthritis]. Vopr Kurortol Fizioter Lech Fiz Kult. 2015 MayJun;92(3):11-13. Russian. doi: 10.17116/kurort2015311-13. PMID: 26285327. 